$\mathrm{E}$

EVALUAR
2017, Vol. 17, No 2.

ISSN 1667-4545

Recuperado de https://revistas.unc.edu.ar/index.php/revaluar

Laboratorio de Evaluación Psicológica y Educativa

Facultad de Psicología - Universidad Nacional de Córdoba

\title{
Análisis Factorial Confirmatorio del IPAM en Escolares de Tercer Curso de Primaria
}

\section{Confirmatory Factor Analysis of IPAM in Third-Grade Students}

\author{
Juan E. Jiménez ${ }^{*}{ }^{1}$, Sara del Cristo de León ${ }^{1}$ \\ 1 - Universidad de La Laguna, España
}

\author{
\begin{tabular}{|l|} 
Introducción \\
Método \\
Resultados \\
Discusión \\
Referencias
\end{tabular}
}

Recibido: 12/07/2017 Revisado: 16/08/2017 Aceptado: 16/08/2017

\section{Resumen}

Este estudio pretende evaluar la estructura factorial del instrumento Indicadores de Progreso de Aprendizaje en Matemáticas (IPAM) mediante la técnica de análisis factorial confirmatorio (AFC). Con este fin, se ha llevado a cabo un estudio longitudinal con una muestra de 234 alumnos de tercer curso de educación primaria de las Islas Canarias, a los que se administró el instrumento IPAM, un instrumento de medición basado en el currículo (CBM, por sus siglas en inglés, curriculum-based measurement), y cuyo principal objetivo es el cribado universal y la evaluación del progreso en el aprendizaje en matemáticas del alumnado de educación primaria. Este instrumento está compuesto por tres medidas paralelas (A, B y C), que pretenden medir una misma estructura latente, el sentido numérico, por medio de la resolución de cinco tareas de fluidez (comparación numérica, operaciones de dos dígitos, series numéricas, operaciones de un dígito y valor de posición). El IPAM fue aplicado en tres momentos diferentes a lo largo del año escolar (i.e., otoño, invierno y primavera) y los resultados del AFC mostraron un buen ajuste del modelo propuesto en los distintos momentos de medida.

Palabras clave: sentido numérico, educación primaria, matemáticas, análisis factorial confirmatorio, medida basada en el currículo

\begin{abstract}
This study has been designed to analyse the factorial structure of IPAM using Confirmatory Factorial Analysis (CFA) techniques. For this purpose, a longitudinal study was carried out with a sample of 234 third-grade elementary students from the Canary Islands, to whom the instrument IPAM (Mathematics Learning Progress Indicators) was administered. IPAM is a curriculum-based measurement (CBM) instrument for universal screening and mathematics learning progress monitoring in elementary grades. It is composed by three parallel measurements (A, B and C) that aim to measure the same latent structure (i.e., number sense) through the assessment of five indicators of basic early math skills using fluency tasks (i.e., magnitude comparison, two-digit operations, missing number, one-digit operations, position value). IPAM was administered three times throughout the school year (i.e., fall, winter, and spring). The model tested showed a good fit at the different moments of measurement.
\end{abstract}

Keywords: number sense, elementary education, mathematics, confirmatory factor analysis, curriculum-based measurement

*Correspondencia a: Juan E. Jiménez. Dirección Postal: Facultad de Psicología, Campus de Guajara, S/N 38200, La Laguna, Islas Canarias. Tel.: +34 922 317545. ejimenez@ull.es

Cómo citar este artículo: Jiménez, J. E., \& De León, S. del C. (2017). Análisis factorial confirmatorio del IPAM en escolares de tercer curso de primaria. Revista Evaluar, 17(2), 81-96. Recuperado de https://revistas.unc.edu.ar/index.php/revaluar

Nota de autor: Este trabajo ha sido posible gracias a la financiación del Plan Nacional I+D+i del Ministerio de Economía y Competitividad, con ref. EDU2012-35098. Investigador Principal: Juan E. Jiménez. 


\section{Introducción}

El sentido numérico ha demostrado ser una habilidad fundamental en el aprendizaje de las matemáticas (Dyson, Jordan, \& Glutting, 2011; Geary, 2013; Jordan, Glutting, \& Ramineni, 2010; Jordan, Kaplan, Ramineni, \& Locuniak, 2009; Kolkman, Kroesbergen, \& Leseman, 2013; Libertus, Fiegenson, \& Halberda, 2011; Lyons \& Beilock, 2011; National Council of Teachers of Mathematics [NCTM], 2000; Park \& Brannon, 2014; Sasanguie, Göbel, Moll, Smets, \& Reynvoet, 2013; Sayers \& Andrews, 2015; Starr, Libertus, \& Brannon, 2013; Toll, Kroesbergen, \& Van Luit, 2016). Este constructo ha sido analizado desde una perspectiva evolutiva y se ha llegado a diferenciar un sentido numérico de naturaleza más informal, previo a la propia adquisición del habla (Dehaene, 2009; Jordan \& Levine, 2009; Libertus et al., 2011; Piazza, 2010), y un sentido numérico de carácter formal, que se va desarrollando por medio de la escolarización (Andrews \& Sayers, 2015; Jordan \& Levine, 2009; National Mathematic Advisory Panel [NMAP], 2008).

Dehaene (2009) propuso que la primera fase del desarrollo del sentido numérico viene "incorporada" en el ser humano, lo que nos permite determinar aproximadamente el número de objetos que hay en una escena, si ese número es mayor o menor que otro, y cómo cambia, por medio de adiciones y sustracciones sencillas, de manera rápida, automática e inconsciente. El sentido numérico evoluciona de manera progresiva y posibilita la adquisición de un sentido numérico simbólico, que nos permite comprender y manipular los números y su representación escrita (Dehaene, 2009). El inicio de la escolarización facilita la formación del sentido numérico fundamental o básico (FoNS, por sus siglas en inglés, Foundational Number Sense), el cual se construye sobre las habilidades subyacentes al sentido numérico prever- bal (Andrews \& Sayers, 2015; Jordan \& Levine, 2009; Sayers \& Andrews, 2015).

Son muchos los autores que han aportado evidencia de los componentes que subyacen al FoNS. En este sentido, Andrews y Sayers (2015) han establecido al menos ocho habilidades básicas: reconocimiento del número, sistema de conteo, relación entre los números y las cantidades que estos representan, discriminación de cantidades, reconocimiento de las distintas representaciones del número, estimación, operaciones aritméticas básicas y patrones numéricos. En consonancia con esta clasificación, otros autores han encontrado que las habilidades vinculadas al conteo, las relaciones entre los números y la resolución de operaciones aritméticas son clave en el desarrollo del sentido numérico (Dyson et al., 2011; Jordan et al., 2010; Jordan \& Levine, 2009). En la misma línea, el NMAP (2008) ha establecido que el sentido numérico supone disponer de habilidades de conteo, discriminación de cantidades, estimación de magnitudes, resolución de operaciones aritméticas sencillas, y comprensión del valor de posición de los dígitos dentro de los números.

Atendiendo a lo expuesto, en este trabajo se han considerado cinco categorías para definir las habilidades subyacentes al sentido numérico: 1) magnitudes numéricas; 2) conteo; 3) línea numérica; 4) valor de posición, y 5) operaciones aritméticas.

\section{¿Cómo medir el sentido numérico en tercer curso de educación primaria?}

Las medidas basadas en el currículo (CBM, por sus siglas en inglés, curriculum based measurement) han demostrado ser adecuadas para establecer diferencias en el desempeño académico entre los estudiantes (Foegen, Jiban, \& Deno, 2007; Gersten et al., 2012; Jitendra, Dupuis, \& 
Zaslofsky, 2014), lo que facilita la detección temprana de estudiantes en riesgo de presentar dificultades de aprendizaje en el área de las matemáticas (Lembke \& Foegen, 2009; Peng et al., 2016; Sasanguie et al. 2013; Sisco-Taylor, Fung, \& Swanson, 2015). Un gran número de herramientas basadas en el sentido numérico han demostrado poseer un potencial adecuado para la evaluación del logro académico en esta área (Andrews \& Sayers, 2015; Jordan et al., 2010; Lembke \& Foegen, 2009; Schneider et al., 2016). No obstante, muchas de las investigaciones llevadas a cabo, centradas en las habilidades básicas que subyacen al sentido numérico (Kim, Shin, \& Lee, 2013; Kolkman et al., 2013; Jiménez \& De León, 2017; Lyons \& Beilock, 2011; Sasanguie et al. 2013) se han enfocado en los primeros cursos de la escolaridad (i.e., educación infantil y primer y segundo curso de educación primaria; Andrews \& Sayers, 2015; Dyson et al., 2011; Foegen et al., 2007; Gersten et al., 2012; Hassinger-Das, Jordan, Glutting, Irwin, \& Dyson, 2014; Jiménez \& De León 2017; Jordan et al., 2010; Lembke \& Foegen, 2009; Libertus, Fiegenson, \& Halberda, 2013; Sasanguie et al., 2013; Starr et al., 2013; Toll et al., 2016), mientras que es menor el número de investigaciones llevadas a cabo en cursos superiores (i.e., tercero, cuarto, quinto $\mathrm{y}$ sexto año de educación primaria; Foegen et al., 2007; Jitendra et al., 2014; Jordan et al., 2010; Lyons, Price, Vaessen, Blomert, \& Ansari, 2014; Namkung \& Fuchs, 2016).

Considerando lo expuesto anteriormente, se ha diseñado la medida Indicadores de Progreso de Aprendizaje en Matemáticas (IPAM; Jiménez \& De León, 2016), que incluye un total de cinco tareas: comparación numérica, secuencias numéricas, operaciones de uno y dos dígitos, y valor de posición.

El objetivo de este trabajo es poner a prueba un modelo hipotético que permita analizar la es- tructura factorial de este instrumento en alumnos de tercer curso de educación primaria. Para ello se empleará la técnica de análisis factorial confirmatorio (AFC), con el fin de conocer en qué medida la varianza asociada a cada una de las tareas que componen la herramienta IPAM (comparación numérica, operaciones de dos dígitos, series numéricas, operaciones de un dígito, y valor de posición) es explicada por un único factor asociado al constructo sentido numérico.

\section{Método \\ Participantes}

La población de este estudio corresponde al alumnado de $3^{\circ}$ curso de educación primaria perteneciente a cinco centros de educación primaria, uno concertado y cuatro públicos, de la provincia de Santa Cruz de Tenerife. La muestra incluyó un total de 234 estudiantes, de los cuales 100 eran niños y 134 eran niñas. La edad promedio de la muestra fue de 8.34, con un rango de edad de 7.33 a 9.75 años.

\section{Instrumentos}

Se utilizó la herramienta Indicadores de Progreso de Aprendizaje en Matemáticas (IPAM; Jiménez \& De León, 2016) diseñada para tercer curso de educación primaria. El IPAM es un instrumento CBM compuesto por tres medidas alternativas o paralelas ( $\mathrm{A}, \mathrm{B}$ y $\mathrm{C}$ ) que han sido diseñadas para medir un mismo constructo (el sentido numérico). Estas medidas se aplican de manera trimestral, en noviembre (Forma A), febrero (Forma $\mathrm{B}$ ), y mayo (Forma $\mathrm{C}$ ) respectivamente. El principal objetivo de este instrumento es el cribado universal y la evaluación del progreso en el aprendizaje del alumnado, mediante la 
evaluación de su fluidez en la resolución de cada tarea. La fluidez es medida contabilizando el número de respuestas correctas que da el alumno en un tiempo dado.

Cada una de las formas está compuesta por cinco tareas, que se aplican de forma consecutiva con una duración máxima de dos minutos por tarea. En la primera tarea, comparación numérica $(\mathrm{CN})$, al estudiante se le presenta una serie de parejas de números y se le solicita rodear aquel que presente mayor magnitud en cada una de las parejas dadas. En la segunda tarea, operaciones de dos dígitos (ODD), se requiere que el estudiante resuelva de forma correcta operaciones de adición, sustracción y multiplicación. En la tercera tarea, secuencias numéricas ( $\mathrm{SN}$ ), se presenta una serie de secuencias compuestas por dos dígitos y una incógnita, la cual el alumno debe completar con el número correspondiente. La cuarta tarea, operaciones de un dígito (OUD), implica la resolución de operaciones básicas de adición, sustracción y multiplicación, en las que se emplean números del 1 al 9. Finalmente, en la quinta tarea, valor de posición (VP), a partir de una representación gráfica dada el estudiante debe determinar el valor posicional de las cifras.

\section{Procedimiento}

Antes de la administración del instrumento, se solicitó autorización a cada centro educativo y a las familias para la realización del estudio. Para llevar a cabo la recogida de información siete licenciados en Psicología y dos licenciados en Psicopedagogía fueron entrenados en la aplicación de la herramienta IPAM.

Las pruebas se aplicaron en tres momentos durante el año escolar (noviembre, febrero y mayo). La aplicación se llevó a cabo de forma colectiva en cada una de las aulas de clase y en horario lectivo, y se informó a los estudiantes del objetivo del estudio.

\section{Análisis de datos}

En este estudio se postula un modelo y se lo evalúa mediante un AFC efectuado sobre los datos empíricos obtenidos a través de las respuestas proporcionadas por los estudiantes en la herramienta IPAM. El modelo propuesto postula la existencia de un único factor de primer orden y, por tanto, que todas las tareas que incluye la herramienta están relacionadas con ese factor. Este modelo se corresponde con una concepción teórica unidimensional del sentido numérico.

En la primera fase hemos formulado la estructura de relaciones entre los indicadores observables y el factor latente. Una vez establecida la estructura del instrumento a nivel conceptual, ésta se postula como la teoría cuya dimensionalidad será validada, con base en los resultados obtenidos.

El segundo paso ha consistido en emplear la metodología de modelado de ecuaciones estructurales (SEM, por sus siglas en inglés, Structural Ecuation Modeling) implementada mediante el paquete LISREL 8.8, con la modalidad de AFC (Jöreskog \& Sörbom, 1996-2001). Este procedimiento consiste en analizar la estructura de covarianza en la base de datos que contiene los cinco indicadores observables (tareas) para obtener evidencia de validez de constructo y poder evaluar si los datos se ajustan al modelo propuesto.

La fase de especificación del modelo incluye una representación gráfica de la estructura teórico-conceptual del instrumento bajo análisis. Por ser el IPAM una herramienta formada por tres medidas, se presenta una representación gráfica de cada una de ellas en el análisis de los resultados en las Figuras 1-3. 
En cada una de estas figuras, el círculo representa el factor latente (constructo) y los óvalos, los cinco indicadores observables (tareas). Las flechas unidireccionales representan las líneas de influencia del factor sobre los respectivos indicadores observables. Estas especificaciones de direccionalidad equivalen a afirmar que cada uno de los indicadores relacionados mide la dimensión a la que en teoría pertenece. Finalmente, las flechas unidireccionales ubicadas a la derecha de los indicadores observables representan los términos de error, que son la influencia de fuentes de variabilidad única (o idiosincrática) asociadas a cada indicador observable.

Se utilizó el Software R (R Core Team, 2013) para el cálculo de estadísticos descriptivos y de los coeficientes de correlación de los resultados obtenidos en las tareas, los cuales se calcularon con el fin de confirmar la ausencia de cualquier efecto de multicolinealidad entre los indicadores. Se calculó la media, desviación típica, máximo, mínimo, asimetría y curtosis para caracterizar los datos de la muestra.

Mediante el Software R y ULLRToolbox (Hernández \& Betancort, 2016), se evaluó la validez factorial de este modelo a través de un AFC. La confiabilidad compuesta (Composite Reliability) y la varianza media extraída (Average Variance Extracted) para cada factor se calcularon según las indicaciones de Fornell y Larcker (1981). La existencia de los valores atípicos se evaluó por la distancia al cuadrado de Mahalanobis $\left(\mathrm{D}^{2}\right)$ y la normalidad de los indicadores se evaluó mediante los coeficientes de asimetría y curtosis, uni y multivariante.

Se utilizó el método de estimación de máxima verosimilitud. La evaluación del método se realizó mediante los siguientes índices: chi-cuadrada $\left(\chi^{2}\right)$, chi cuadrada sobre los grados de libertad $\left(\chi^{2} / d f\right)$, índice de ajuste comparativo (CFI), bondad de ajuste del modelo (GFI), y error cuadrático medio de aproximación (RMSEA), P [RMSEA $\leq .05]$.

\section{Resultados}

A la hora de exponer los resultados es importante recordar que la herramienta IPAM está compuesta por tres formas aplicadas consecutivamente a intervalos regulares. De este modo, se expondrán en primer lugar los resultados correspondientes a la Forma A, en segundo lugar, los resultados de la Forma B y, finalmente, los resultados de la Forma C.

\section{IPAM Forma A}

En la Tabla 1 se presentan los análisis estadísticos descriptivos de cada una de las tareas que componen el IPAM (Forma A). Se muestran los valores máximo y mínimo, medias, desviación estándar, asimetría y curtosis para cada tarea. Un primer análisis de estos datos muestra que los valores de asimetría y curtosis son cercanos a cero. De este modo, se evidencia una distribución normal y una dispersión satisfactoria de los resultados, con la excepción de valor de posición, que tiende a valores más altos en relación al promedio, aunque se mantiene dentro de los rangos aceptables para estos estadísticos. Al observar los valores de asimetría y curtosis de todas las variables del estudio se puede corroborar que no existen problemas con la distribución de los datos, puesto que los valores absolutos de estos índices no exceden los valores 3.0 y 10.0 respectivamente (Kline, 2005). Las desviaciones en las puntuaciones estándar se sitúan entre 9.98 y 2.76 . 
Tabla 1

Estadísticos descriptivos de IPAM Forma A.

\begin{tabular}{lcccccc}
\hline & Mínimo & Máximo & Media & $\begin{array}{c}\text { Desviación } \\
\text { estándar }\end{array}$ & Asimetría & Curtosis \\
\hline CN & 4 & 63 & 39.35 & 9.98 & -.40 & 1.23 \\
ODD & .0 & 16 & 5.52 & 2.76 & .46 & .82 \\
SN & .0 & 22 & 8.59 & 5.65 & .18 & -1.02 \\
OUD & .0 & 40 & 13.52 & 6.17 & .30 & -.21 \\
VP & .0 & 16 & 6.89 & 3.36 & 1.14 & 2.98 \\
\hline
\end{tabular}

Nota. $\mathrm{CN}=$ Comparación numérica, $\mathrm{ODD}=$ Operaciones de dos dígitos, $\mathrm{SN}=$ Series numéricas, $\mathrm{OUD}=$ Operaciones de un dígito, $\mathrm{VP}=$ Valor de posición .

Tabla 2

Coeficientes de correlación entre las tareas de IPAM Forma A.

\begin{tabular}{lccccc}
\hline & CN & ODD & SN & OUD & VP \\
\hline CN & 1.00 & $.33^{* * *}$ & $.47^{* * *}$ & $.43^{* * *}$ & $.48^{* * *}$ \\
ODD & & 1.00 & $.51^{* * *}$ & $.60^{* * *}$ & $.44^{* * *}$ \\
SN & & & 1.00 & $.55^{* * *}$ & $.54^{* * *}$ \\
OUD & & & & 1.00 & $.47^{* * *}$ \\
VP & & & & & 1.00 \\
\hline
\end{tabular}

Nota. $\mathrm{CN}=$ Comparación numérica, $\mathrm{ODD}=$ Operaciones de dos dígitos, $\mathrm{SN}=$ Series numéricas, $\mathrm{OUD}=$ Operaciones de un dígito, $\mathrm{VP}=\mathrm{Valor}$ de posición . $* * * p<.001$.

La matriz de correlación de los resultados obtenidos en las diferentes tareas que componen el IPAM (Forma A) se presenta en la Tabla 2. Los resultados obtenidos muestran correlaciones estadísticamente significativas entre todos los indicadores.

En primer lugar, el AFC da como resultado una representación de las relaciones estructurales observadas en la matriz derivada. Los valores que representan las flechas unidireccionales son las líneas de influencia entre el factor latente y los indicadores observables y se interpretan como coeficientes de regresión multivariada. Estos han sido calculados por el programa, al analizar la matriz de covarianza derivada de los indicadores observables. Así también, se han calculado los coeficientes de regresión entre el factor latente y los indicadores observables. El primer valor que se encuentra es .61, el cual corresponde a la influencia del factor latente sentido numérico sobre el indicador observable comparación numérica $(\mathrm{CN})$. El coeficiente de regresión presenta signo positivo, lo que evidencia una relación directa entre el factor latente y el indicador observable. Esto implica que cuando el factor latente aumenta una unidad, la variable observada $\mathrm{CN}$ aumenta en una proporción de .61. La misma interpretación cabe para las relaciones existentes entre el factor latente y el resto de los indicadores observables, ya que se encontró un coeficiente de .63 para operaciones con dos dígitos (ODD), un coeficiente de .78 para series numéricas (SN), un coeficiente de .68 para operaciones con un dígito (OUD), y un coeficiente de .70 para valor de posición (VP) (ver Figura 1). 


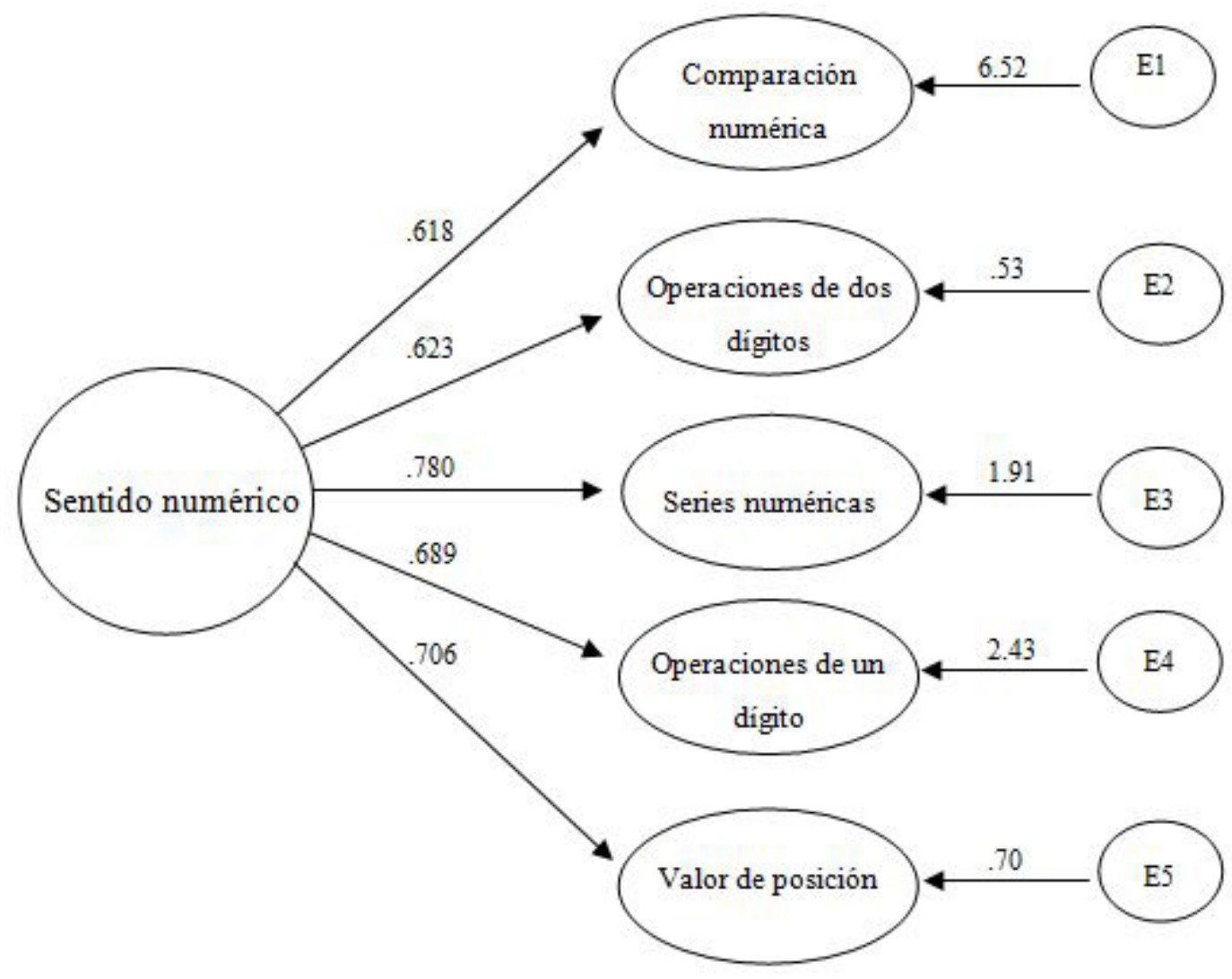

Figura 1

Modelo de un factor general. IPAM Forma A

La evaluación preliminar del modelo permitió verificar en los coeficientes derivados el sentido positivo esperado de acuerdo con la teoría empleada en la configuración del modelo de medida.

Posteriormente, se analizó el nivel de ajuste del modelo propuesto con los datos. En primer lugar, se obtuvo un valor de $\chi^{2}$ igual a $4.28(p=.37)$, lo que implica que el modelo y los resultados se ajustan entre sí. Para confirmar este resultado, se procedió a la evaluación del ajuste del modelo mediante los índices de bondad de ajuste. Los resultados indicaron un buen ajuste del modelo a los datos empíricos $\left(\mathrm{SRMR}=.02 ; \chi^{2}=4.28 ; d f=\right.$ $4 ; p=.37$; NFI $=.99 ; \mathrm{CFI}=.99 ; \mathrm{GFI}=.99 ; \mathrm{AGFI}$ $=.97 ; \mathrm{RMSEA}=.02 ; \mathrm{IC}=.00-.10)$.

Para conseguir un ajuste adecuado del modelo fue necesario liberar la covarianza de error entre los indicadores observables ODD y OUD. Una posible característica común compartida por estos indicadores y no explicada por la variable latente estaría relacionada con la capacidad para inhibir respuestas competitivas y seleccionar la respuesta más adecuada (LeFevre et al., 2013; Lemaire \& Lecacheur, 2011; Lucangeli, Tressoldi, Bendotti, Bonanomi, \& Siegel, 2003). Por ejemplo, cuando un niño resuelve una suma, puede utilizar varias estrategias, como contar desde el primer número de la operación en adelante, contar desde el mayor, o recuperar el resultado de un hecho numérico ya almacenado en la memoria (Siegler \& Araya, 2005). Por otro lado, cuando el niño ya ha asimilado determinados hechos numéricos, pero aún está aprendiendo otros, los que ya conoce se activan al intentar resolver una 
Tabla 3

Estadísticos descriptivos de IPAM Forma B.

\begin{tabular}{lcccccc}
\hline & Mínimo & Máximo & Media & $\begin{array}{c}\text { Desviación } \\
\text { estándar }\end{array}$ & Asimetría & Curtosis \\
\hline CN & 11 & 64 & 47.29 & 10.53 & -.41 & .20 \\
ODD & 0 & 21 & 7.41 & 3.23 & .73 & 1.66 \\
SN & 0 & 27 & 10.62 & 5.94 & .13 & -.70 \\
OUD & 0 & 45 & 15.76 & 7.74 & .91 & 1.49 \\
VP & 0 & 20 & 7.26 & 4.05 & .45 & .13 \\
\hline
\end{tabular}

Nota. $\mathrm{CN}=$ Comparación numérica, $\mathrm{ODD}=$ Operaciones de dos dígitos, $\mathrm{SN}=$ Series numéricas, $\mathrm{OUD}=$ Operaciones de un dígito, $\mathrm{VP}=$ Valor de posición .

Tabla 4

Coeficientes de correlación entre las tareas de IPAM Forma B.

\begin{tabular}{lccccc}
\hline & CN & ODD & SN & OUD & VP \\
\hline CN & 1.00 & $.36^{* * *}$ & $.46^{* * *}$ & $.43^{* * *}$ & $.46^{* * *}$ \\
ODD & & 1.00 & $.58^{* * *}$ & $.69^{* * *}$ & $.50^{* * *}$ \\
SN & & & 1.00 & $.66^{* * *}$ & $.66^{* * *}$ \\
OUD & & & & 1.00 & $.63^{* * *}$ \\
VP & & & & & 1.00 \\
\hline
\end{tabular}

Nota. $\mathrm{CN}=$ Comparación numérica, $\mathrm{ODD}=$ Operaciones de dos dígitos, $\mathrm{SN}=$ Series numéricas, $\mathrm{OUD}=$ Operaciones de un dígito, $\mathrm{VP}=$ Valor de posición . *** $p<.001$.

determinada operación. Por ello, es necesario que inhiba las respuestas que se activan automáticamente por estar asociadas a un determinado hecho numérico conocido, pero que son incorrectas. Se trataría, por ejemplo, de inhibir la respuesta " 8 " ante la pregunta "¿cuánto es 4 x 4?”, ya que "8” es la respuesta automática que se activa, por tener asimilado que " $4+4$ es igual a 8 " (Cragg \& Gilmore, 2014; Siegler \& Araya, 2005).

Tanto en OUD como en ODD parece evidente que la atención ejecutiva, a la hora de inhibir determinada información en favor de otra más adecuada, así como de seleccionar la estrategia que mejor se ajusta a la demanda de la tarea, puede jugar un papel importante en la resolución de estas tareas, ya que el estudiante debe resolver correctamente sumas, restas y multiplicaciones en el menor tiempo posible, lo que le obliga a se- leccionar una estrategia que le permita actuar de forma rápida y eficaz, es decir, con fluidez. En este sentido, LeFevre et al. (2013) encontraron que la atención ejecutiva es un factor crítico en la resolución de operaciones aritméticas de manera fluida y en el acceso a los hechos numéricos.

\section{IPAM Forma B}

En la Tabla 3 se presentan los análisis estadísticos descriptivos de cada una de las tareas que componen el IPAM (Forma B). Se muestran los valores máximo y mínimo, medias, desviación estándar, asimetría y curtosis para cada tarea. Un primer análisis de estos datos muestra una dispersión satisfactoria para todas las tareas. Los coeficientes de curtosis se aproximan a cero, lo que 


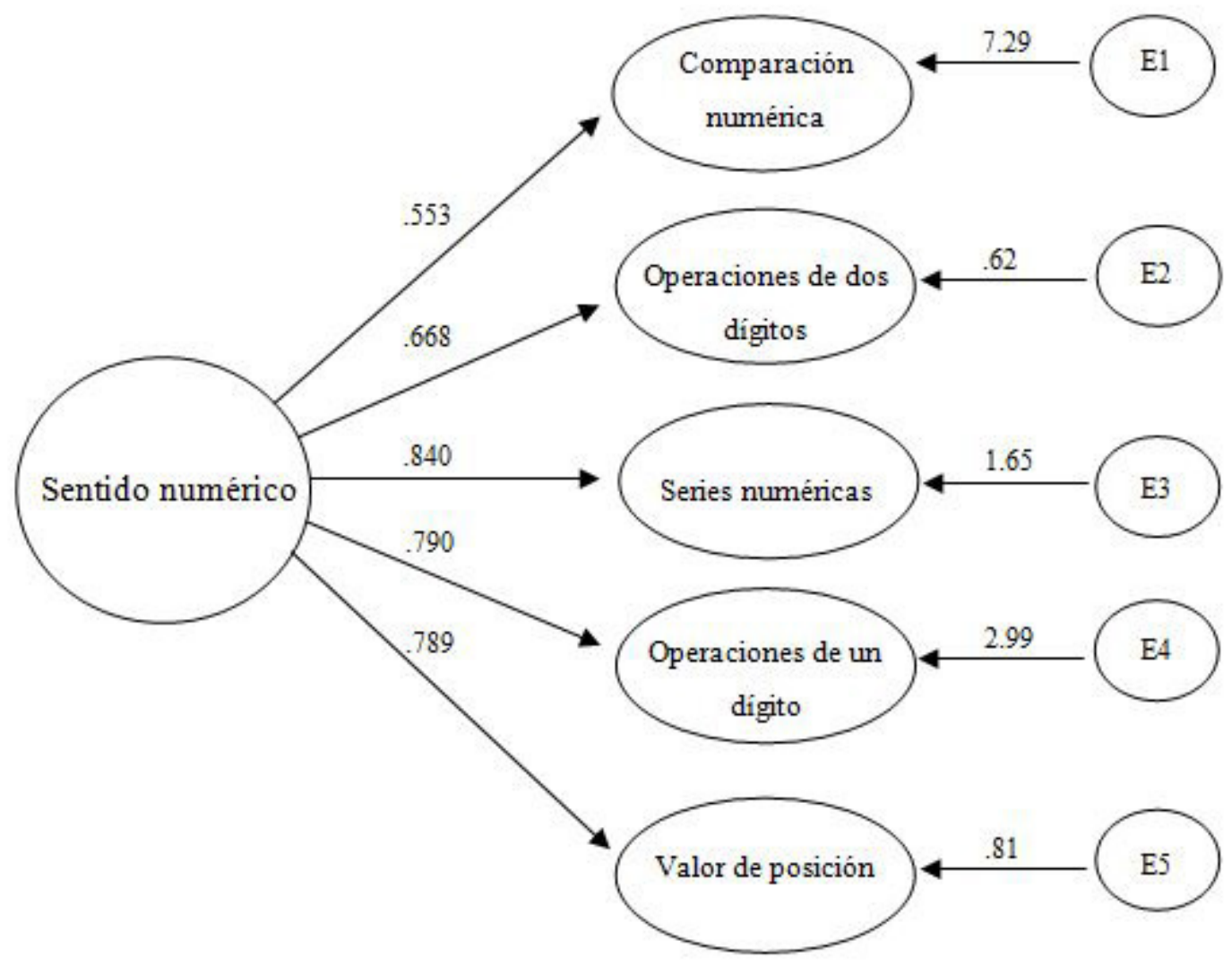

Figura 2

Modelo de un factor general. IPAM Forma B

indica una distribución normal de los resultados (Kline, 2005). Las desviaciones en las puntuaciones estándar se sitúan entre 10.53 y 3.23.

La matriz de correlación de los resultados obtenidos en las diferentes tareas que componen el IPAM (Forma B) se presenta en la Tabla 4. Los resultados obtenidos muestran correlaciones estadísticamente significativas entre todos los indicadores.

Los resultados obtenidos en el AFC de la Forma B del IPAM se han interpretado de la misma manera que aquellos de la Forma A, en cuanto a la representación de las relaciones estructurales observadas en la matriz derivada y a los coeficientes de regresión multivariada. De este modo, el sentido numérico funciona como factor latente y las tareas son sus indicadores observables. En este caso, $\mathrm{CN}$ presenta un coeficiente de regresión de .55, ODD un coeficiente de .66, SN un coeficiente de .84, OUD un coeficiente de $79 \mathrm{y}$ VP presenta un coeficiente de .78 (ver Figura 2).

Así, la evaluación preliminar del modelo permitió verificar para los coeficientes derivados el sentido positivo esperado de acuerdo con la teoría empleada en la configuración del modelo de medida.

Posteriormente, se analizó el nivel de ajuste del modelo propuesto con los datos. El valor de $\chi^{2}=2.06(p=.72)$ implica que el modelo $\mathrm{y}$ los resultados se ajustan entre sí. Los índices de bondad de ajuste confirman el ajuste del modelo $\left(\mathrm{SRMR}=.01 ; \chi^{2}=2.06 ; d f=4 ; p=.72 ; \mathrm{NFI}=\right.$ $.99 ; \mathrm{CFI}=1 ; \mathrm{GFI}=.99 ; \mathrm{AGFI}=.98 ; \mathrm{RMSEA}=$ $0 ; \mathrm{IC}=0-.07)$. 
Tabla 5

Estadísticos descriptivos de IPAM Forma C.

\begin{tabular}{lcccccc}
\hline & Mínimo & Máximo & Media & $\begin{array}{c}\text { Desviación } \\
\text { estándar }\end{array}$ & Asimetría & Curtosis \\
\hline CN & 22 & 64 & 52.31 & 9.55 & -.78 & .22 \\
ODD & 0 & 27 & 7.27 & 4.00 & 1.30 & 3.58 \\
SN & 0 & 28 & 12.19 & 6.26 & -.09 & -.53 \\
OUD & 1 & 45 & 21.05 & 8,57 & .44 & .32 \\
VP & 0 & 44 & 9.97 & 6.13 & 1.65 & 5.65 \\
\hline
\end{tabular}

Nota. $\mathrm{CN}=$ Comparación numérica, $\mathrm{ODD}=$ Operaciones de dos dígitos, $\mathrm{SN}=$ Series numéricas, $\mathrm{OUD}=$ Operaciones de un dígito, $\mathrm{VP}=$ Valor de posición .

Tabla 6

Coeficientes de correlación entre las tareas de IPAM Forma C.

\begin{tabular}{lccccc}
\hline & CN & ODD & SN & OUD & VP \\
\hline CN & 1.00 & $.47^{* * *}$ & $.50^{* * *}$ & $.58^{* * *}$ & $.41^{* * *}$ \\
ODD & & 1.00 & $.60^{* * *}$ & $.73^{* * *}$ & $.38^{* * *}$ \\
SN & & & 1.00 & $.66^{* * *}$ & $.49^{* * *}$ \\
OUD & & & & 1.00 & $.43^{* * *}$ \\
VP & & & & & 1.00 \\
\hline
\end{tabular}

Nota. $\mathrm{CN}=$ Comparación numérica, $\mathrm{ODD}=$ Operaciones de dos dígitos, $\mathrm{SN}=$ Series numéricas, $\mathrm{OUD}=$ Operaciones de un dígito, $\mathrm{VP}=\mathrm{Valor}$ de posición . $* * * p<.001$.

Para que este modelo se ajustase a los datos fue también necesario liberar la covarianza de error existente entre ODD y OUD. Esta covarianza, al igual que en la forma A del IPAM, estaría explicada por la capacidad de inhibir distintas estrategias que se activarían cuando se trata de resolver una determinada operación (LeFevre et al., 2013; Lemaire \& Lecacheur, 2011; Lucangeli et al., 2003).

\section{IPAM Forma C}

En la Tabla 5 presentamos los análisis estadísticos descriptivos de cada una de las tareas que componen el IPAM (Forma C). Se muestran los valores máximo y mínimo, medias, desviación estándar, asimetría y curtosis para cada tarea. Un primer análisis de estos datos muestra que los resultados presentan una dispersión satisfactoria para todas las tareas salvo para ODD y VP. Los valores de asimetría y curtosis son cercanos a cero, lo que indica una distribución normal de los resultados, con la excepción de ODD y VP, los cuales presentan valores por encima de la unidad/uno. A pesar de ello, podemos observar que no existen problemas con la distribución de los datos, puesto que los valores absolutos de estos estadísticos no exceden los valores 3.0 y 10.0 respectivamente (Kline, 2005). Las desviaciones en las puntuaciones estándar se sitúan entre 9.55 y 4.00 .

La matriz de correlación de los resultados obtenidos en las diferentes tareas que componen el IPAM (Forma C) se presenta en la Tabla 6. Los resultados obtenidos muestran correlaciones estadísticamente significativas entre todos los indicadores. 


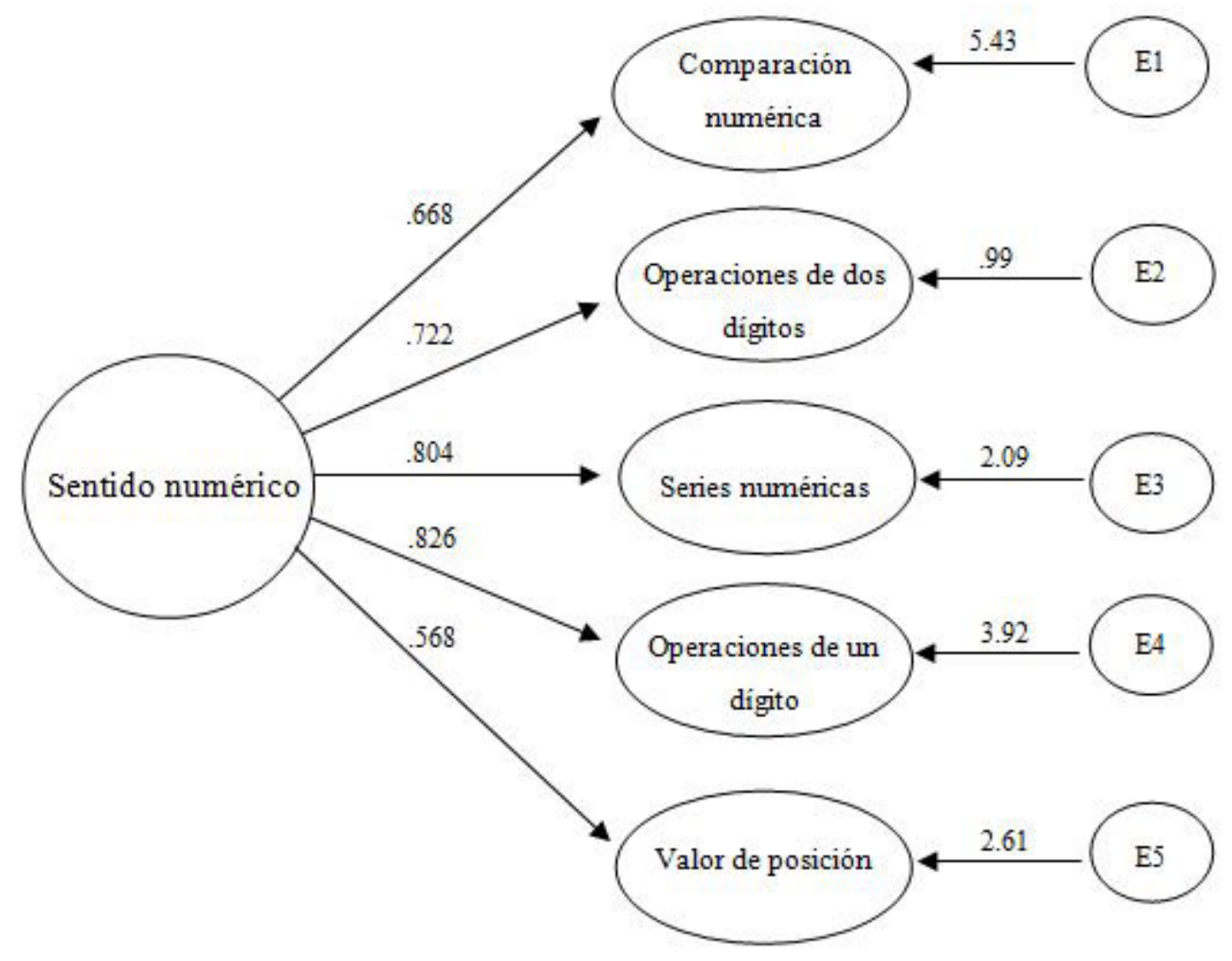

Figura 3

Modelo de un factor general. IPAM Forma C

Los resultados obtenidos en el AFC de la Forma $\mathrm{C}$ del IPAM se han interpretado de la misma manera que aquellos de la Forma A, en lo que se refiere a la representación de las relaciones estructurales observadas en la matriz derivada y a los coeficientes de regresión multivariada. De este modo, el sentido numérico funciona como factor latente y las tareas son sus indicadores observables. En este caso, $\mathrm{CN}$ presenta un coeficiente de regresión de .66, ODD un coeficiente de .72, SN un coeficiente de .80 , OUD un coeficiente de $.82 \mathrm{y}$ VP presenta un coeficiente de .56 (Ver Figura 3).

Así, la evaluación preliminar del modelo permite verificar que los coeficientes derivados presentan el sentido positivo esperado de acuerdo con la teoría empleada en la configuración del modelo de medida.
Posteriormente, se analizó el nivel de ajuste del modelo propuesto con los datos. El valor de $\chi^{2}=7.77(p=.101)$ implica que el modelo y los resultados se ajustan entre sí. Los índices obtenidos a través del análisis de la bondad de ajuste confirman el ajuste del modelo $\left(\mathrm{SRMR}=.02 ; \chi^{2}\right.$ $=7.77 ; d f=4 ; p=.10 ; \mathrm{NFI}=.98 ; \mathrm{CFI}=1$; GFI $=.99 ; \mathrm{AGFI}=.95 ; \mathrm{RMSEA}=.06 ; \mathrm{IC}=0-.13$ ).

Para que este modelo se ajustase a los datos fue necesario liberar la covarianza de error existente entre ODD y OUD. Esta covarianza, al igual que en las formas A y B del IPAM, estaría explicada por la capacidad para inhibir respuestas competitivas y seleccionar la respuesta más adecuada (LeFevre et al., 2013; Lemaire \& Lecacheur, 2011; Lucangeli et al., 2003). Esta habilidad, dependiente de las funciones ejecuti- 
vas (es decir, inhibición y flexibilidad cognitiva o atención ejecutiva), permite a los estudiantes resolver operaciones aritméticas con mayor fluidez (LeFevre et al., 2013).

\section{Discusión}

En este trabajo se ha pretendido poner a prueba un modelo hipotético para verificar la estructura factorial del instrumento IPAM, diseñado para medir el sentido numérico, en tercer curso de educación primaria, mediante la técnica de análisis factorial confirmatorio (AFC). Los datos obtenidos y el análisis de la estructura factorial según la técnica AFC permiten verificar un buen ajuste del modelo propuesto con los datos empíricos. Así, se observa cómo el factor latente sentido numérico explica cada uno de los indicadores observables (comparación numérica, operaciones de dos dígitos, secuencias numéricas, operaciones de un dígito, valor de posición), para cada uno de los momentos de medida (noviembre, febrero y mayo).

Los resultados obtenidos se ajustan a lo prescrito por la teoría en cuanto a la definición del constructo y las habilidades que lo conforman, incluidas las habilidades tempranas típicamente asociadas con el desarrollo del sentido numérico (Andrews \& Sayers, 2015; Dyson et al., 2011; Jordan et al., 2010; Jordan \& Levine, 2009; NMAP, 2008; Sayers \& Andrews, 2015).

Varios trabajos han sido desarrollados con el fin de diseñar herramientas efectivas, basadas en el sentido numérico, que permitan evaluar el rendimiento en matemáticas de los estudiantes de tercer curso de educación primaria. En este sentido, Lyons et al. (2014) analizaron la relación existente entre las habilidades de procesamiento simbólico del número y las habilidades aritméticas en alumnos de primero a sexto curso de edu- cación primaria. Los resultados mostraron que las habilidades de las que disponían los niños en procesamiento numérico explicaban la mayoría de la varianza asociada a las habilidades aritméticas en estos cursos. Schneider et al. (2016) encontraron que la habilidad para procesar magnitudes (cálculo mental y habilidades matemáticas tempranas) estaba altamente asociada con las competencias matemáticas de las que disponían los estudiantes, y era una de las habilidades fundamentales para el diseño de herramientas de cribaje y la intervención tanto con niños como con adultos. Jordan et al. (2010) encontraron que el sentido numérico era un fuerte predictor del logro en matemáticas tanto en primer curso como en tercer curso de educación primaria. Otros estudios apoyan los resultados obtenidos por estos trabajos, por haber encontrado que las habilidades que conforman el sentido numérico juegan un papel importante en el desarrollo y adquisición de habilidades matemáticas o aritméticas más complejas (De Smedt, Verschaffel, \& Ghesquière, 2009; Holloway \& Ansari, 2009; Piazza, 2010).

La principal aportación de la presente investigación ha sido la de contrastar un modelo basado en la teoría, donde hemos podido constatar que el sentido numérico influye en más de una medida observada y que da cuenta de las correlaciones entre estas medidas observadas (i.e., comparación numérica, operaciones de dos dígitos, secuencias numéricas, operaciones de un dígito y valor de posición). Estas variables observadas están intercorrelacionadas porque comparten un factor causal común, esto es, se encuentran influenciadas por el mismo constructo subyacente: el sentido numérico.

Los mismos resultados fueron encontrados por Jiménez y De León (2017) para primer curso de educación primaria, lo que sugiere que las habilidades básicas subyacentes al sentido numérico se mantienen estables desde primer hasta tercer 
curso de educación primaria. No obstante, se ha observado que en primer curso el factor latente sentido numérico influye principalmente en los indicadores observables operaciones de un dígito y operaciones de dos dígitos (Jiménez \& De León, 2017); mientras que en tercer curso el factor latente sentido numérico influye principalmente en los indicadores observables series numéricas, operaciones de un dígito y valor de posición.

Una de las posibles limitaciones del presente estudio está relacionada con el conjunto de tareas que han sido seleccionadas, ya que no podemos descartar que otras tareas, por ejemplo la resolución de problemas verbales (ver Jordan et al., 2010; Sisco-Taylor et al., 2015), puedan ser igualmente válidas para la medición del sentido numérico.

Se encontró una covarianza de error estable en los tres momentos de medida (noviembre, febrero y mayo) entre las tareas OUD y ODD. Es posible que en estas edades una característica compartida por esos indicadores, y no explicada por la variable latente, esté relacionada con la capacidad para inhibir respuestas competitivas y seleccionar la respuesta más adecuada cuando se trata de resolver una operación aritmética (LeFevre et al., 2013; Lemaire \& Lecacheur, 2011; Lucangeli et al., 2003). En este sentido, Lemaire y Lecacheur (2011) encontraron que las funciones ejecutivas y la edad se correlacionaban de manera significativa con la capacidad de la que disponen los niños para discriminar, entre distintas estrategias, aquella que es más adecuada para resolver una determinada tarea aritmética. En la misma línea, en un estudio anterior realizado por Fuchs et al. (2006) se encontró que la atención correlacionaba tanto con las operaciones numéricas básicas (operaciones de un dígito) como con aquellas más complejas (operaciones de dos dígitos o más).

Concluimos que el IPAM presenta una adecuada validez de constructo ya que permite captar la capacidad de sentido numérico en estudiantes de tercer curso de educación primaria. A la vista de la revisión de estudios, se pone de manifiesto que esta capacidad estaría en la base de las dificultades que pueden experimentar algunos niños durante los primeros cursos de educación formal en el área de las matemáticas. Líneas futuras de investigación han de centrarse en analizar la sensibilidad y especificidad del IPAM para la detección de estudiantes en riesgo de presentar dificultades específicas de aprendizaje en matemáticas, así como su validez a la hora de evaluar el progreso en el aprendizaje.

\section{Referencias}

Andrews, P., \& Sayers, J. (2015). Identifying opportunities for grade one children to acquire foundational number sense: Developing a framework for cross cultural classroom analyses. Early Childhood Education Journal, 43(4), 257-267. doi: 10.1007/ s10643-014-0653-6

Cragg, L., \& Gilmore, C. (2014). Skills underlying mathematics: The role of executive function in the development of mathematics proficiency. Trends in Neuroscience and Education, 3(2), 63-68. doi: 10.1016/j.tine.2013.12.001

Dehaene, S. (2009). Origins of mathematical intuitions. The case of arithmetic. Annals of the New York Academy of Sciences, 1156(1), 232-259. doi: 10.1111/j.1749-6632.2009.04469.x

De Smedt, B., Verschaffel, L., \& Ghesquière, P. (2009). The predictive value of numerical magnitude comparison for individual differences in mathematics achievement. Journal of Experimental Child Psychology, 103(4), 469-479. doi: 10.1016/j.jecp.2009.01.010

Dyson, N. I., Jordan, N. C., \& Glutting, J. (2011). A number sense intervention for low-income kindergartners at risk for mathematics difficulties. Journal of Learning Disabilities, 46(2), 166-181. doi: 
$10.1177 / 0022219411410233$

Foegen, A., Jiban, C., \& Deno, S. (2007). Progress monitoring measures in mathematics. A review of the literature. The Journal of Special Education, 41(2), 121-139. doi: 10.1177/00224669070410020101

Fornell, C., \& Larcker, D. F. (1981). Evaluating structural equation models with unobservable variables and measurement error. Journal of Marketing Research 18, 39-50. doi: 10.2307/3151312

Fuchs, L. S., Fuchs, D., Compton, D. L., Powel, S. R., Seethaler, P. M., Capizzi, A. M. ... Fletcher, J. M. (2006). The cognitive correlates of third-grade skill in arithmetic, algorithmic computation, and arithmetic word problems. Journal of Educational Psychology, 98(1), 29-43. doi: 10.1037/0022-0663.98.1.29

Geary, D. C. (2013). Early foundations for mathematics learning and their relations to learning disabilities. Current Directions in Psychological Science, 22(1), 23-27. doi: 10.1177/0963721412469398

Gersten, R., Clarke, B., Jordan, N. C., Newman-Gonchar, R., Haymond, K., \& Wilkins, C. (2012). Universal screening in mathematics for the primary grades: Beginnings of a research base. Council for Exceptional Children, 78(4), 423-445. doi: 10.1177/001440291207800403

Hernández, J. A., \& Betancort, M. (2016). ULLRToolbox. Disponible en https://sites.google.com/site/ ullrtoolbox

Hassinger-Das, B., Jordan, N. C., Glutting, J., Irwin, C., \& Dyson, N. (2014). Domain-general mediators of the relation between kindergarten number sense and first-grade mathematics achievement. Journal of Experimental Child Psychology, 118, 78-92. doi: 10.1016/j.jecp.2013.09.008

Holloway, I. D., \& Ansari, D. (2009). Mapping numerical magnitudes onto symbols: The numerical distance effect and individual differences in children's mathematics achievement. Journal of Experimental Child Psychology, 103(1), 17-29. doi: 10.1016/j. jecp.2008.04.001

Jiménez, J. E., \& De León, S. (2016). Indicadores de
Progreso de Aprendizaje en Matemáticas (IPAM). Universidad de La Laguna. Manuscrito sin publicar. Jiménez, J. E., \& De León, S. (2017). Análisis factorial confirmatorio de Indicadores de Progreso de Aprendizaje en Matemáticas (IPAM) en escolares de primer curso de primaria. European Journal of Investigation in Health, Psychology and Education, 7, 31-45. Recuperado de https://formacionasunivep.com/ejihpe/index.php/ejihpe

Jitendra, A. K., Dupuis, D. N., \& Zaslofsky, A. F. (2014). Curriculum-based measurement and standards-based mathematics: Monitoring the arithmetic word problem-solving performance of third-grade students at risk for mathematics difficulties. Learning Disability Quarterly, 37(4), 241-251. doi: 10.1177/0731948713516766

Jordan, N. C., Gutting, J., \& Ramineni, C. (2010). The importance of number sense to mathematics achievement in first and third grades. Learning and Individual Differences, 20, 82-88. doi: 10.1016/j. lindif.2009.07.004

Jordan, N. C., Kaplan, D., Ramineni, C., \& Locuniak, M. N. (2009). Early math matters: Kindergarten number competence and later mathematics outcomes. Developmental Psychology, 45(3), 850-867. doi: $10.1037 / \mathrm{a} 0014939$

Jordan, N. C., \& Levine, S. C. (2009). Socioeconomic variation, number competence, and mathematics learning difficulties in young children. Developmental Disabilities Research Reviews, 15(1), 60-68. doi: 10.1002/ddrr.46

Jöreskog, K., \& Sörbom, D. (1996-2001). LISREL 8: User's Reference Guide. Illinois: Scientific Software International, Lincolnwood.

Kim, D., Shin, J., \& Lee, K. (2013). Exploring latent class based on growth rates in number sense ability. Asia Pacific Education Review, 14(3), 445-453, doi: 10.1007/s12564-013-9274-9

Kline, R. B. (2005). Principles and practice of structural equation modeling ( $2^{\mathrm{a}}$ ed.). Nueva York, NY: Guilford Press. 
Kolkman, M. E., Kroesbergen, E. H., \& Leseman, P. P. M. (2013). Early numerical development and the role of non-symbolic and symbolic skills. Learning and Instruction, 25, 95-103. doi: 10.1016/j. learninstruc.2012.12.001

LeFevre, J., Berrigan, L., Vendetti, C., Kamawar, D., Bisanz, J., Skwarchuck, S. ... Smith-Chant, B. (2013). The role of executive attention in the acquisition of mathematical skills for children in grades 2 through 4 . Journal of Experimental Child Psychology, 114(2), 243-261. doi: 10.1016/j.jecp.2012.10.005

Lemaire, P., \& Lecacheur, M. (2011). Age-related changes in children's executive functions and strategy selection: A study in computational estimation. Cognitive Development, 26, 282-294. doi: 10.1016/j. cogdev.2011.01.002

Lembke, E., \& Foegen, A. (2009). Identifying early numeracy indicators for kindergarten and first-grade students. Learning Disabilities Research \& Practice, 24(1), 12-20. doi: 10.1111/j.1540-5826.2008.01273.x

Libertus, M. E., Fiegenson, L., \& Halberda, J. (2011). Preschool acuity of the approximate number system correlates with school math ability. Developmental Science, 14(6), 1292-1300. doi: 10.1111/j.1467-7687.2011.01080.x

Libertus, M. E., Fiegenson, L., \& Halberda, J. (2013). Is approximate number precision a stable predictor of math ability? Learning and Individual Differences, 25, 126-133. doi: 10.1016/j.lindif.2013.02.001

Lucangeli, D., Tressoldi, P. E., Bendotti, M., Bonanomi, M., \& Siegel, L. S. (2003). Effective strategies for mental and written arithmetic calculation from the third to the fifth grade. Educational Psychology, 23(5), 507520. doi: 10.1080/0144341032000123769

Lyons, I. M., \& Beilock, S. L. (2011). Numerical ordering ability mediates the relation between number-sense and arithmetic competence. Cognition, 121(2), 256261. doi: 10.1016/j.cognition.2011.07.009

Lyons, I. M., Price, G. R., Vaessen, A., Blomert, L., \& Ansari, D. (2014). Numerical predictors of arithmetic success in grades 1-6. Developmental Science,
17(5), 714-726. doi: 10.1111/desc.12152

Namkung, J. M., \& Fuchs, L. S. (2016). Cognitive predictors of calculations and number line estimation with whole numbers and fractions among at-risk students. Journal of Educational Psychology, 108(2), 214-228. doi: $10.1037 /$ edu0000055

National Council of Teachers of Mathematics (2000). Principles and standards for school mathematics. Reston, VA: NCATE.

National Mathematics Advisory Panel (2008). Foundations for Success: The Final Report of the National Mathematics Advisory Panel. Washington, DC: Department of Education.

Park, J., \& Brannon, E. M. (2014). Improving arithmetic performance with number sense training: An investigation of underlying mechanism. Cognition, 133(1), 188-200. doi: 10.1016/j.cognition.2014.06.011

Peng, P., Namkung, J. M., Fuchs, D., Fuchs, L. S., Patton, S., Yen, L. ... Hamlett, C. (2016). A longitudinal study on predictors of early calculation development among young children at risk for learning difficulties. Journal of Experimental Child Psychology, 152, 221-241. doi: 10.1016/j.jecp.2016.07.017

Piazza, M. (2010). Neurocognitive start-up tools for symbolic number representations. Trends in Cognitive Sciences, 14(12), 542-551. doi: 10.1016/j. tics.2010.09.008

R Core Team (2013). R: A language and environment for statistical computing. R Foundation for Statistical Computing, Vienna, Austria. Recuperado de http:// Www.R-project.org

Sasanguie, D., Göbel, S. M., Moll, K., Smets, K., \& Reynvoet, B. (2013). Approximate number sense, symbolic number processing, or number-space mappings: What underlies mathematics achievement? Journal of Experimental Child Psychology, 114(3), 418-431. doi: 10.1016/j.jecp.2012.10.012

Sayers, J., \& Andrews, P. (2015). Foundational number sense: Summarising the development of an analytical framework. En K. Krainer \& N. Vondrová (Eds.), Ninth Congress of the European Society for Research 
in Mathematics Education (CERME9) (pp. 361337). Praga: Charles University in Prague, Faculty of Education.

Schneider, M., Beeres, K., Coban, L., Merz, S., Schmidt, S. S., Stricker, J. ... De Smedt, B. (2016). Associations of non-symbolic and symbolic numerical magnitude processing with mathematical competence: A meta-analysis. Developmental Science, 20(3), 1-16. doi: 10.1111/desc. 12372

Siegler, R., \& Araya, R. (2005). A computational model of conscious and unconscious strategy discovery. Advances in Child Development and Behavior, 33, 1-42. doi: 10.1016/S0065-2407(05)80003-5

Sisco-Taylor, D., Fung, W., \& Swanson, H. L. (2015). Do curriculum-based measures predict performance on word-problem-solving measures? Assessment for Effective Intervention, 40(3), 131-142. doi: 10.1177/1534508414556504

Starr, A., Libertus, M. E., \& Brannon, E. M. (2013). Number sense in infancy predicts mathematical abilities in childhood. Proceedings of the National Academy of Science, 110(45), 18116-18120. doi: 10.1073/ pnas. 1302751110

Toll, S. W. M., Kroesbergen, E. H., \& Van Luit, J. E. H. (2016). Visual working memory and number sense: Testing the double deficit hypothesis in mathematics. British Journal of Educational Psychology, 86(3), 429-445. doi: 10.1111/bjep.12116 